\title{
Practice Spotlight: Denise Sprague
}

\author{
Denise Sprague, BScPharm, ACPR, PharmD \\ Clinical Pharmacy Specialist - \\ Infectious Diseases \\ Kelowna General Hospital \\ Kelowna, British Columbia
}

$\mathrm{K}_{\mathrm{m}}^{\mathrm{c}}$ elowna General Hospital is a 344-bed tertiary referral hospital within the Interior Health Authority of British Columbia, which offers all major medical specialties to about 300000 people in the Okanagan Valley. Infectious Diseases, a consultative service at Kelowna General Hospital, comprises one infectious diseases physician and one pharmacist. Denise Sprague currently holds the pharmacist position within this service. She and the physician work closely with other health care professionals, including the clinical microbiologist, the microbiology laboratory technicians, the home IV therapy pharmacist, the Vascular Access Program nurses, and infection control practitioners, among others. The Infectious Diseases service typically receives 5 to 10 new inpatient consults per week (primarily adults), with ongoing follow-up of 15 to 25 inpatients.

The position currently held by Dr Sprague was created in 2008 by Interior Health Regional Pharmacy Services and is funded by Kelowna General Hospital's Pharmacy Services. It was created in response to an identified need for a full-time clinical pharmacist to provide pharmacist activities in the busy Infectious Diseases service and in response to increasing requests from all Interior Health pharmacists for education about infectious diseases pharmacotherapy. The infectious diseases pharmacist will also train residents in infectious diseases when Interior Health initiates a hospital pharmacy residency program in 2011. Dr Sprague became the first infectious diseases pharmacist at Kelowna General Hospital in 2008, after completing her Doctor of Pharmacy at the University of British Columbia. She was actively involved in the development of the unique roles and responsibilities of the new position. Not only was she the first pharmacist dedicated to the inpatient Infectious Diseases service, but she was also the first clinical pharmacist at the hospital to practise as a consultant, rather than a ward-based clinical pharmacist. Before starting her practice, Dr Sprague met with pharmacy staff, the infectious diseases physician, and other key health care professionals working in this area to obtain input on how her position could be integrated into both the pharmacy and infectious diseases teams.

The clinical pharmacist works Monday to Friday, her main responsibility being to provide pharmaceutical care to all inpatients seen by the infectious diseases physician. The initial visit includes verification of the patient's medication, vaccination, and allergy history. She is responsible for ensuring that inpatients with infectious diseases receive any required vaccinations. She is also responsible for daily monitoring of antimicrobial efficacy and tolerability, including signs and symptoms, diagnostic results, microbiology culture results, laboratory values, and pharmacokinetic monitoring. She works closely with the infectious diseases physician and other members of the health care team to ensure that every patient is receiving the most effective and safest antimicrobial therapy, taking into account all patient-specific factors. Patient education and seamless care activities are also an integral part of this pharmacist's role in patient care.

The infectious diseases pharmacist is a local, regional, and provincial resource. She participates on local and regional infection control committees, the Interior Health Antimicrobial Subcommittee, and the outbreak management teams for Kelowna General Hospital, as well as local, regional, and provincial pandemic planning committees. As the only infectious diseases pharmacist in the Interior Health authority, Dr Sprague provides education and acts as a drug information resource for pharmacists and other health care professionals across the health authority. She offers infectious diseases rotations for pharmacy undergraduate students, residents, and PharmD students. She also teaches infectious diseases pharmacotherapy in the undergraduate and PharmD programs at the University of British Columbia. Additional nonclinical activities include collaborating with other health care professionals to develop protocols and programs to facilitate appropriate use of antimicrobials. When opportunities arise, she also participates in drug-use evaluation and practice-based research at Kelowna General Hospital.

As the role of the infectious diseases pharmacist expands in the future, the use of pharmacy technicians may be considered to assist with vaccine and medication reconciliation. The British Columbia Pharmacy Association now offers a certification program for pharmacists to administer injections. Given the important role that the infectious diseases pharmacist plays in immunization, this specialized certification should be considered to further expand the pharmacist's role. 